\title{
Introduction of Modeling Complex Management Systems using Fuzzy Cognitive Map
}

\author{
Miklos F. Hatwagner \\ Dept. of Information Technology \\ Adrienn Buruzs \\ Dept. of Environmental Engineering \\ Andras Torma \\ Dept. of Environmental Engineering \\ Laszlo T. Koczy \\ Dept. of Information Technology \\ Szechenyi Istvan University, Hungary
}

\begin{abstract}
Fuzzy Cognitive Maps (FCM) have found favor in a variety of theoretical and applied contexts that span the hard and soft sciences. Given the utility and flexibility of the method, coupled with the broad appeal of FCM to a variety of scientific disciplines, FCM have been appropriated in many different ways and, depending on the academic discipline in which it has been applied, used to draw a range of conclusions about the belief systems of individuals and groups. In scenario planning, causal mapping has long been used as a means to elicit the worldviews of multiple experts, facilitate discussion, and challenge and improve mental models. Large and complex causal maps, however, are difficult to analyze. The strength of FCM approach lies in its capacity not only to comprehensively model qualitative knowledge which dominates strategic decision making but also to stimulate and evaluate several alternative way of using IT in order to improve organizational performance. This approach introduces computational modeling, as well as it supports scenarios development and simulations. In this paper the authors focus on the investigation of two possible applications: waste management system and stakeholder management system. The common features of these systems are that both systems are complex and comprehensive.
\end{abstract}

Keywords-Fuzzy Cognitive Maps, Simulation, Integrated Waste Management Systems, Stakeholder Relationship Management

\section{INTRODUCTION}

Modeling dynamic systems can be hard in a computational sense and many quantitative techniques exist. Well-understood systems may be open to any of the mathematical programming techniques of operations study. First, developing the model usually requires a big deal of effort and specialized knowledge outside the area of interest. Secondly, systems involving important feedback may be nonlinear, in which case a quantitative model may not be possible [1].

This paper presents Fuzzy Cognitive Maps as an approach in modeling the behavior and operation of complex systems. This technique is the fusion of the advances of the fuzzy logic and cognitive maps theories, they are fuzzy weighted directed graphs with feedback that create models that emulate the behavior of complex decision processes using fuzzy causal relations.

Fuzzy Cognitive Maps are fuzzy structures that strongly resemble neural networks, and they have powerful and farreaching consequences as a mathematical tool for modeling complex systems.
The purpose of this article is to suggest the use of FCM as an alternative approach to existing strategic planning models used in different fields of management. The article suggests that FCM can be a useful tool to facilitate creativity and synergy. There is a wealth of literature from the fields of cognitive science, psychology, and systems science that discusses the use of individuals' knowledge structures as representations or abstractions of real world phenomena [2].

First the description and the methodology that this theory suggests is examined, also some ideas for using this approach in the management area, and then the usage of this tool is described. The application of this approach in the field of system management might contribute to the progress of more intelligent and more objective evaluation of the systems. Fuzzy Cognitive Maps have been fruitfully used in decision making and simulation of complex situation and analysis.

\section{FUZZY COGNITIVE MAPS (FCM)}

Decision makers usually face serious difficulties when approaching significant, real-world dynamic systems. Such systems are composed of a number of dynamic concepts or 
actors which are interrelated in complex ways, usually including feedback links which propagate influences in complicated chains [3].

In the development of the FCM, in the first step of the design process the number and features of constituting factors are determined by the relevant literature. These concepts are supposed to be combined all together in a single system, with mutual interactions.

Modern technological systems are complex and they are usually comprised of a large number of interacting and coupling entities that are called subsystems and/or components. These systems have nonlinear behavior and cannot simply be derived from summation of analyzed individual component behavior [4]. Feedback mechanisms are important in the analysis of vulnerability and resilience of social-economictechnical systems. But how to evaluate systems with direct feedbacks has been a great challenge. FCM was derived from the fusion of fuzzy logic and theory of cognitive maps. Kosko [5] developed the fuzzy signed directed graphs with feedback in order to represent knowledge in a comprehensive way. Since the FCM is formed for a selected system by determining the concepts and their relationships, it is possible to quantitatively simulate the system considering its parameters. It has to be noted however, that a FCM is suitable for short term time series analysis and prediction. A FCM is a dynamic modeling tool in which the resolution of the system representation can be increased by applying a further mapping. The resulting fuzzy model can be used to analyze, simulate, and test the influence of parameters and predict the behavior of the system [6].

According to [6], the design of a FCM is a process that heavily relies on the input from experts and/or stakeholders. This methodology extracts the knowledge from the stakeholders and exploits their experience on the system's model and behavior. A FCM is fairly simple and easy to understand for the participants. With the use of a participatory process it should be ensured that different interests are used to build up synergies as well as partnerships and hence find sustainable solutions as a joint decision [7]. Even though, the cognitive nature of a FCM makes it inevitably a subjective representation of the system. The model is not arbitrary as it is built carefully and reflexively with stakeholders [8].

On the basis of a FCM's development, during the first step in the designing process, the number and features of concepts are determined by a group of experts. After the identification of the main factors affecting the topic under investigation, each stakeholder is asked to describe the existence and type of the

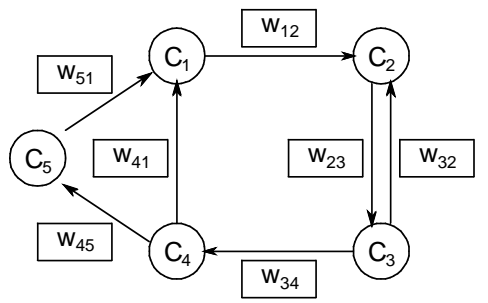

Fig. 1. Example of a simple Fuzzy Cognitive Map causal relationships among these factors and then assesses the strength of these causal relationships using a predetermined scale, capable to describe any kind of relationship between two factors, positive and negative.

Starting from the primary elements of a FCM, the $i$ th concept denotes a state, a procedure, an event, a variable or an input of the system and is represented by $C_{i}(i=1,2, \ldots, n)$. Another component of a FCM is the directed edge which connects the concepts $i$ and $j$. Each edge includes a weight $w_{i j}$ which represents the causality between concepts $C_{i}$ and $C_{j}$. The values of the concepts are within the range $[0,1]$, while the values of the weights belong to the interval $[-1,1]$. A positive value of the weight $w_{i j}$ indicates that an increase (decrease) in the value of concept $C_{i}$ results to an increment (decrement) of the concept's value $C_{j}$. Similarly, a negative weight $w_{i j}$ indicates that an increase (decrease) in the value of concept $C_{i}$ results to a decrement (increment) of the concept's value $C_{j}$, while a zero weight denotes the absence of relationship between $C_{i}$ and $C_{j}$ (Fig. 1). Considering the interrelations between the concepts of a FCM, the corresponding adjacency matrix can easily be formed. Usually it is accepted that causality is not self reflexive, i.e., a concept cannot cause itself, which means that the weight matrix always has ' 0 -s' in its diagonal [9]. Otherwise the component values may be unstable.

The description of the inference mechanism, which represents the behavior of the physical system, lies in the interpretation of FCM's mathematical formulation. After the initialization of the FCM and the determination of concept activation values by experts, concepts are ready to interact. As it is obvious, the activation of a concept influences the values of concepts that are connected to it. At each step of interaction (simulation step), every concept acquires a new value that is calculated according to equations (Equation 1 and 2) and the interaction between concepts continues until a fix equilibrium is reached; a limit cycle is reached; or a chaotic behavior is observed [10].

The mathematical description of our FCM system is a simple loop:

Where $V_{k}$ is the state $k$ of the system; $N$ is the matrix of the system which contains the weight $w_{i, j}$, and

where $\lambda>0$ determines the steepness of the of the continuous function $f$.

The FCM is a very convenient and simple tool for modeling complex systems. It is rather popular due to its simplicity and user friendliness. According to [11], human experts are generally rather subjective and can handle only relatively simple networks therefore there is an urgent need to develop methods for automated generation of FCM models. 
An FCM is a fuzzy graph structure representing causal reasoning. Causality is represented here as a fuzzy relation of causal concepts. The FCM may be used for dynamic modeling of systems. The FCM approach uses nodes corresponding to the factors and edges for their interactions, to model different aspects in the behavior of the system. These factors interact with each other in the FCM simulation, presenting the dynamics of the original system [4]. The FCM has been described as the combination of neural networks and fuzzy logic. Thus, learning techniques and algorithms can be borrowed and utilized in order to train the FCM and adjust the weights of its interconnections [12].

\section{INTEGRATED WASTE MANAGEMENT SYSTEMS}

The treatment of waste became one of the most important assignments of today. Several cultural, social, industrial and financial phenomena are responsible for the increasing amount and the more and more diverse types of waste. Many problems of waste processing can be avoided by the consistent usage of source control and appropriate treatment of waste. This way the ratio of reused and recycled waste can be increased. The goal of sustainable waste management is to decrease the amount of waste placed at landfills by e.g. recycling and composting. This part of the paper describes and models the Integrated Waste Management Systems (IWMS) on regional level applying Fuzzy Cognitive Maps (FCM).

During the research process the main factors, the key drivers of a sustainable IWMS were identified at first. After the throughout study of literature [16-21] it can be stated now that a wide-ranging consensus took shape in this topic. The factors are the following: environmental, economic, social, institutional, legal and technical. These factors determine the operation and behavior of such a system. This approach was accepted by the authors and taken into consideration as wellfounded.

During the last decades several kinds of models were developed [22] to monitor the processes of waste management, to support decision making and to foresee the possible future outcomes of these decisions. Models based on expert knowledge can help to solve several environmental problems, including IWMS, too. The applied method makes possible to extract the cumulative knowledge and exploit the experiences of stakeholders in order to model the system and its behavior.

The authors' intention was to model an IWMS using FCM. An FCM needs the description of causal relationships among the factors. The factors were already identified using the

TABLE II. THE INITIAL DRAFT OF THE CONNECTION MATRIX

\begin{tabular}{|l||l|l|l|l|l|l|}
\hline & \multicolumn{1}{|c|}{ C1 } & \multicolumn{1}{|c|}{ C2 } & \multicolumn{1}{|c|}{ C3 } & \multicolumn{1}{c|}{ C4 } & \multicolumn{1}{c|}{ C5 } & \multicolumn{1}{c|}{ C6 } \\
\hline \hline C1 & 0 & 0.8 & 0.6 & 0.6 & 0.4 & 0.4 \\
\hline C2 & 0.6 & 0 & 0.6 & 0.6 & 0.4 & 0.4 \\
\hline C3 & 0.8 & 0.6 & 0 & 0.6 & 0.4 & 0.4 \\
\hline C4 & 0.4 & 0.6 & 0.4 & 0 & 0.4 & 0.4 \\
\hline C5 & 0.6 & 0.6 & 0.4 & 0.6 & 0 & 0.6 \\
\hline C6 & 0.4 & 0.4 & 0.4 & 0.4 & 0.4 & 0 \\
\hline
\end{tabular}

TABLE I. THE INITIAL STATE VECTOR

\begin{tabular}{|c|c|}
\hline Factor & $\mathbf{t}_{\mathbf{0}}$ \\
\hline \hline C1 & 0.20 \\
\hline C2 & 0.15 \\
\hline C3 & 0.10 \\
\hline C4 & 0.10 \\
\hline C5 & 0.10 \\
\hline C6 & 0.10 \\
\hline
\end{tabular}

literature, as it was mentioned before, but the strengths and directions of interactions were unknown. In order to solve this problem, a questionnaire was created. All stakeholders participated in this on-line survey was asked to describe the relationships (directions and weights) between factors using a predetermined simple scale. The applicable values could be both positive and negative. A guideline was also created to support the work of the stakeholders, i.e. to describe the terms of concepts and the goal and basics of research. Finally, 75 different connection matrices were created on the basis of the stakeholders' answers. They were merged into a single but representative map (see Table 1) by averaging source matrices. The factors were denoted in the following way: C1) technical factor, C2) environmental factor, C3) economic factor, C4) social factor, C5) legal factor and C6) institutional factor.

The description of causal relationships among factors is not enough to begin the simulation of IWMS using FCM, however. The other input of the model is the initial state vector of the factors. The data originates from literature [14-21] and represented by real numbers between 0 and 1 (see Table 2).

Several simulations were made with different $\lambda$ (threshold function parameter) values, but it affects only the values of factors at the end of simulation, not the order of them. This can be an important issue in practice, however, because an unfavorably selected $\lambda$ results in almost equal factor values that makes really hard to determine the real order of factors. The presented simulation contained 10 iterative steps, but in most cases less iteration would be enough. Fig. 2 shows that factor values converged really fast to their final, stable values. The values of factors in the last simulation step and their order are presented in Table 3 . The members of the set containing the

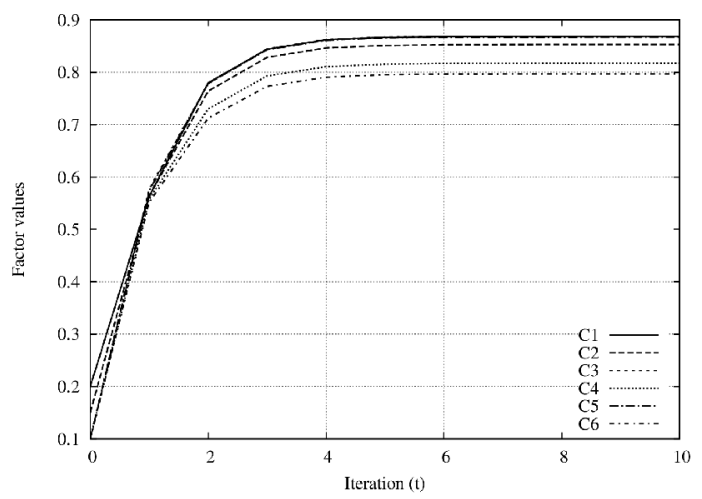

Fig. 2. The model simulation with $\lambda=0.8$ 
ICIT 2015 The $7^{\text {th }}$ International Conference on Information Technology

doi:10.15849/icit.2015.0092 C ICIT 2015 (http://icit.zuj.edu.jo/ICIT15)

TABLE III. THE ORDER AND FINAL VALUES OF VECTORS

\begin{tabular}{|l|c|c|}
\hline Factor & Order & Value \\
\hline \hline C1 & 2 & 0.8685 \\
\hline C2 & 4 & 0.8530 \\
\hline C3 & 1 & 0.8688 \\
\hline C4 & 5 & 0.8173 \\
\hline C5 & 3 & 0.8675 \\
\hline C6 & 6 & 0.7970 \\
\hline
\end{tabular}

highest factor values are $\mathrm{C} 1$ (technical factor), C3 (economic factor) and C5 (legal factor). Their values are almost the same. They are followed by factor C2 (environmental factor), C4 (social factor) and C6 (institutional factor) is the last in order.

An important result of the simulation is that the order of factors defines the priority of factors in the IWMS on regional level. The technical factor defines how and what materials are managed, treated and disposed of. This field covers the attributes of collection, transfer and treatment systems, e.g. organic material treatment, thermal treatment, materials recovery and final disposal.

The economic issues (available funding, system costs and revenues, etc.) have practically the same importance. The third component is the relevant legislation, e.g. prescriptive or enabling legislation; EU, national, and municipal level legislation; legal definition of municipal solid waste. The next factor in the order of importance is the environmental factor. It covers e.g. the livability of the settlements, the pollution in different areas. The following element in the list is the social factor. The main issue here is to minimize the risks to public health, adapting the system to the local demands and requirements and to willingness and ability to pay. The final element of the list is the institutional factor. It includes the fields of accountability, stakeholder involvement, transparency and professionalism.

It must be emphasized again that the validity of the results and their applicability in practice depends on the input data. Because the data is collected from a wide range of well-known experts, we are convinced that it is usable to plan or establish new IWMSs at least in a more or less closed geographical area, even if it always could contain subjective convictions.

\section{STAKEHOLDER RELATIONSHIP MANAGEMENT}

Authors investigated the applicability of FCM method to analyse the interconnections between the main factors of Stakeholder Management System. The investigation showed practical usability of the method for the desired purpose.

Stakeholders influence the operation and the decisions of an organization, but they play also a determinative role during a project, a program or even during various activities. The influence made by stakeholders can be very different. All of the stakeholders are actors who are linked with the organization. These actors can be identified by their interest regarding the operation and decisions. Beside that also the attitude of the stakeholders is determinative for this group of actors. Stakeholders can influence the success of the activity in diverse ways, the influence made by them can be in definite cases absolute decisively. Stakeholder management has the aim to deal with stakeholder issues and so it can contribute to the successful operation or to an effective completion of a project. Grouping and identifying the stakeholders by definite parameters is the baseline for all further measures [23]. Wellfounded management of stakeholders contributes to the success of the company and long-term sustainability of the organization [24].

Every organization has interested parties also from intern and from extern. Different categories of stakeholders can be identified such as e.g. professional associations, other companies, shareholders, authorities, employees, or even the customers.

The connection of stakeholders with the organization is two sided: on the one hand they have great information demand about the operation, on the other hand they are influencing the operation. It is not extraordinary that they are communicating and cooperating with each other. Other effect of the stakeholder's activity is that they can form the circumstances of the operation.

Establishing an effective stakeholder management system needs deeper knowledge about the interested parties. This knowledge helps the decision makers to set up the main elements of a management approach, which can contribute to the more effective operation. The most important topic is to get a detailed picture. For that there is a need of deeper assessing the stakeholder structure of an organization. Identifying the main actors is very important but the priority ranking of them plays also a determinative role. The need of effectivity necessitates the priority ranking of the stakeholders because

organizations have limited resources to deal with that issues and that's why it is essential to know the most significant ones.

The commonly used methods of stakeholder analysis used by companies are mainly inquiry techniques or checklistsurveys. With them decision makers can get a more accurate level of information about the stakeholders or even about the organizational attitude and activities regarding management processes in the organization.

These techniques are mainly suitable to outline the structure of stakeholders in a static way. With the help of these methods the experts are not able to get information about the dynamics within the system and the interconnections between the main drivers.

The authors investigated in former research the opportunity of the dynamic modeling of a mapped stakeholder system [25]. The aim of these research was to develop a stable methodology for that. Analyzing the interconnections between the driver elements and the causality as well as the weights of them can help in better understanding of a SRMS.

The research stated that the Fuzzy Cognitive Map methodology $(\mathrm{FCM})$ is suitable for this modelling purpose. For 
the analysis the authors used expert based data on identifying factors of the SRMS.

Traditionally stakeholder analysis is made in two steps. The purposes of this phase of the analysis are the following: (a) to identify the stakeholders; (b) to analyze the relationship with them [26].

The first purpose can be reached by using different management techniques (e.g.: brainstorming of the experts). For the second purpose there are different methods available. Different matrix approaches are used frequently for this analysis. These matrix approaches traditionally investigate two different dimensions, namely the degree of involvement of the stakeholder (from low to high) or their type of influence (opposing or supporting).

This two-dimensional approach of the interconnections between the interested parties gives sufficient information for setting up suitable management strategies. The picture resulting from such static analysis is shows the attitudes of the actors in a definite time moment. The nature of these connections, the casual correspondences of the system and its cross cutting connections are however hidden.

Researches investigating stakeholder management in respect of mainly on the ideal strategies set up on the basis of the two dimensional approach, the concrete activities and applicable management techniques in connection with them.

Stakeholders are differentiated usually by their attitudes. Fig. 3. shows a classic representation of stakeholder groups with different attitudes. Letters from $A$ to $\mathrm{J}$ are representing hypothetic stakeholders of an organization. The main strategy in connection with the stakeholders is to manage the group with the greatest influence and with the greatest interest. It means that the focus area of the management activities is the upper right section of Fig. 3.

Current research does not face with the analysis of causal relations between the stakeholders and the characteristics of this system is not in the focus.

The SRMS gives the frames and the main strategic ways of the management actions mentioned before. Effective solution of the problems regarding stakeholder management can be defined also with the help of the SRMS. Other benefit of such an approach, that this gives the possibility of standardized solutions. Knowing the interactive connections between these main driving elements and the dynamics of such connections gives a detailed picture about the whole system.
TABLE IV. IDENTIFIED CAUSAL CONNECTIONS BETWEEN MAIN CONCEPTS (EXAMPLE OF HUNGARY)

\begin{tabular}{|l|l|l|l|l|l|l|l|l|l|l|l|}
\hline & & & & & & & & & & & \\
& & & & & & & & & \\
& & & & & & & & & & & \\
\hline C1 & 0.9 & 0 & 2 & 0 & 6 & 3 & 2 & 9 & 10 & 10 & 8 \\
\hline C2 & 0.5 & 10 & 0 & 2 & 9 & 8 & 8 & 2 & 7 & 8 & 4 \\
\hline C3 & 0.6 & 5 & 8 & 0 & 9 & 7 & 6 & 6 & 3 & 4 & 6 \\
\hline C4 & 0.9 & 6 & 0 & 8 & 0 & 9 & 10 & 4 & 2 & 3 & 3 \\
\hline C5 & 0.6 & 7 & 4 & 3 & -1 & 0 & 8 & 9 & 8 & 9 & 8 \\
\hline C6 & 0.8 & 4 & 1 & 3 & 4 & 2 & 0 & 3 & 9 & 10 & 7 \\
\hline C7 & 0.7 & 0 & 8 & -2 & 3 & 4 & 4 & 0 & 3 & 3 & 10 \\
\hline C8 & 0.9 & 3 & 6 & 0 & 4 & 2 & 2 & 0 & 0 & 9 & 1 \\
\hline C9 & 1.0 & 0 & 0 & 0 & 1 & 0 & 1 & 0 & 6 & 0 & 0 \\
\hline C10 & 0.4 & 6 & 7 & 1 & 5 & 1 & 3 & 10 & 3 & 4 & 0 \\
\hline
\end{tabular}

The authors investigated the applicability of FCM to model the interconnections between the main criteria of the SRMS and to make conclusions for the application of effective methods to manage the regarding issues.

As the first step the main driver elements of a SRMS were defined. These elements have the biggest influence on the operation efficiency of a SRMS and so they are subject of the management investigations. For this step results of scientific examinations and notions of practitioner business managers was used. The 10 main identified categories were the following: $\underline{\mathrm{C} 1}$ : importance of the stakeholder management; $\underline{\mathrm{C} 2}$ : allocation of resources; $\underline{\mathrm{C} 3}$ : involvement of employees; $\underline{\mathrm{C} 4}$ : organization culture; $\underline{\mathrm{C} 5}$ : internal regulations; $\underline{\mathrm{C} 6}$ : organizational strategy, policy; $\underline{\mathrm{C} 7}$ : internal expectations; $\underline{\mathrm{C} 8}$ : external expectations; $\underline{C}$ : external regulatory instruments; C10: activity of internal stakeholder parties. The categories were also broken down into 48 subcategories to get in the future more appropriate information.

The possible causality and the weights of the connections were measured by interviewing company and scientific experts in Hungary and in Lithuania. The results of the investigation were used as one input data for the FCM-model.

The first detailed analysis was made by using the Hungarian results, where representatives of the private sector and researchers of this field were asked (more than 15 representatives of different business sectors - e.g.: machine manufacturing, service sector and governmental sector). 
ICIT 2015 The $7^{\text {th }}$ International Conference on Information Technology

doi:10.15849/icit.2015.0092 C ICIT 2015 (http://icit.zuj.edu.jo/ICIT15)

TABLE VI. VALUES OF FACTORS WITH OPTIMAL LAMBDA $(\lambda=0.664)$

\begin{tabular}{|c|c|}
\hline Factor & Value \\
\hline $\mathrm{C} 1$ & 0.941 \\
\hline $\mathrm{C} 2$ & 0.967 \\
\hline $\mathrm{C} 3$ & 0.962 \\
\hline $\mathrm{C} 4$ & 0.938 \\
\hline $\mathrm{C} 5$ & 0.958 \\
\hline $\mathrm{C} 6$ & 0.915 \\
\hline $\mathrm{C} 7$ & 0.877 \\
\hline $\mathrm{C} 8$ & 0.817 \\
\hline $\mathrm{C} 9$ & 0.610 \\
\hline $\mathrm{C} 10$ & 0.913 \\
\hline
\end{tabular}

The results were averaged and can be seen in Table 4 (values are varying between $[-10 ; 10]$ ).

For the first model the authors used a fix connection. The initial weighting of the factors were also obtained by expert questioning (see in the second column of Table 4). This information describes the initial state vector of the factors.

Table 5 shows the results of the analysis, namely the priority order of the concepts (with $\lambda=1$; from the most determinative one to the less). The most determinative driver of the SRMS is the availability of the resources (C2) for stakeholder issues. The next two factors both are internal drivers, namely: involvement of employees (C3) and the state of internal regulations within the organization (C5). As fourth and fifth the importance of this topic $(\mathrm{C} 1)$ and the organizational culture $(\mathrm{C} 4)$ are playing important role. The strategy and policy of the organization (C6) influences only the sixth important role. The last four drivers are the activity of internal parties $(\mathrm{C} 10)$, the internal expectations (C7) regarding SRMS, in connection with it the external expectations (C8) and the regulatory instruments of external parties (C9). This ranking gives sufficient information to propose new management strategies for stakeholder issues.

Simulations were made by using different $\lambda$ parameter

TABLE V. PRIORITY LIST OF THE CONCEPTS ON THE BASIS OF MODEL RESULTS (HUNGARIAN VALUES; WITH $\lambda=1$ )

\begin{tabular}{|l|l|}
\hline \multicolumn{2}{|c|}{ Ranking Hungarian values } \\
\hline $\mathrm{C} 2$ & 0.9956 \\
\hline $\mathrm{C} 3$ & 0.9944 \\
\hline $\mathrm{C} 5$ & 0.9937 \\
\hline $\mathrm{C} 1$ & 0.9893 \\
\hline $\mathrm{C} 4$ & 0.9870 \\
\hline $\mathrm{C} 6$ & 0.9794 \\
\hline $\mathrm{C} 10$ & 0.9777 \\
\hline $\mathrm{C} 7$ & 0.9585 \\
\hline $\mathrm{C} 8$ & 0.9162 \\
\hline $\mathrm{C} 9$ & 0.6784 \\
\hline
\end{tabular}

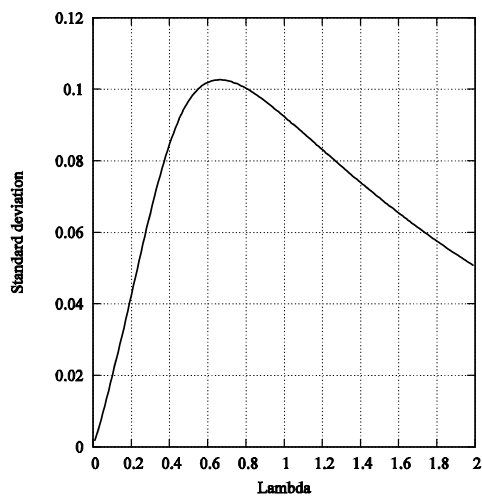

Fig. 4. Value of standard deviation with different $\lambda$ values

(threshold function parameter). The results showed that the data were inconclusive showing almost the same factor value for different drivers. These values made the practical evaluation practically impossible. Several empirical attempts were made in [27] to find an appropriate value for $\lambda$, but the more thorough definition and analysis of this parameter was lacking until now.

Authors looked for the maximal spread out value of them because factor values can be then easier differentiated. The spread will be quantified in the rule of the standard deviation function borrowed from statistics. Different $\lambda$ values result in different factor values. The standard deviation of factors calculated with different $\lambda$ values is depicted by Fig. 4 . The maximum of the standard deviation is 0.103 at $\lambda=0.664$. This result was calculated numerically with the Golden Section Search algorithm, a well-known and rather simple kind of line search methods. Using the optimal $\lambda$ value authors remodeled the causal interconnections. The stable values are listed in Table 6.

Authors used a novel approach to calculate stable FCM values for analyzing management problems. The presented method makes possible to achieve the most easily interpretable simulation results at the cost of executing a computationally inexpensive local search algorithm.

\section{FURTHER RESEARCH}

Our intention is to validate the developed models by experts of the fields. The expected results of these investigations may help to determine the essential steps towards solving these complex problems on the long term and obtain techniques for the sustainability and long term maintenance of the systems.

\section{ACKNOWLEDGMENT}

The authors would like to thank to TÁMOP-4.2.2.A11/1/KONV-2012-0012, TÁMOP-4.1.1.C-12/1/KONV-20120017, to the Hungarian Scientific Research Fund (OTKA) K105529 and K108405 for the support of the research.

\section{REFERENCES}


[1] C. Buche, P. Chevaillier, A. Nédélec, M. Parenthoën, and J. Tisseau. 2010. Fuzzy cognitive maps for the simulation of individual adaptive behaviors. Comput. Animat. Virtual Worlds 21, 6 (November 2010), 573-587. DOI=10.1002/cav.363 http://dx.doi.org/10.1002/cav.363

[2] E. I. Papageorgiou (ed.), Fuzzy Cognitive Maps for Applied Sciences and Engineering, 29, Intelligent Systems Reference Library 54, DOI: 10.1007/978-3-642-39739-4_2, C Springer-Verlag Berlin Heidelberg 2014

[3] J. P. Carvalho, J. A. B. Tomé, Rule Based Fuzzy Cognitive Maps Qualitative Systems Dynamics IFSA-EUSFLAT 2009 Lisbon, Portugal

[4] Stylos, D. and Groumpos, P. P. (2004). Modelling Complex Systems Using Fuzzy Cognitive Maps. IEEE Transactions on Systems, Man, and Cybernetics - Part A: Systems and Humans, 34(1): 155-162.

[5] Kosko, B. (1986). Fuzzy Cognitive Maps. Int. J. of Man-Machine Studies, 24(1): 65-75.

[6] Papageorgiou, E. and Kontogianni, A. (2012). Using Fuzzy Cognitive Mapping in Environmental Decision Making and Management: A Methodological Primer and an Application. Int. Perspectives on Global Environmental Change, S. Young (ed.), ISBN: 978-953-307-815-1, InTech, doi: 10.5772/29375

[7] Malena, C. (2004). Strategic Partnership: Challenges and Best Practices in the Management and Governance of Multi-Stakeholder Partnerships Involving UN and Civil Society Actors. Background paper prepared by for the Multi-Stakeholder Workshop on Partnerships and UN-Civil Society Relations, Pocantico, New York

[8] Isak, K. G. Q., Wildenberg, M., Adamescu, M., Skov, F., De Blust, G. and Varjopuro, R. (2009). A Long-Term Biodiversity, Ecosystem and Awareness Research Network Manual for Applying Fuzzy Cognitive Mapping - Experiences from ALTER-Net. Project no. GOCE-CT-2003505298, ALTER-Net Deliverable type: Report, WPR6-2009-02 Deliverable 4.R6.D2.

[9] Carvalho, J. P. (2010). On the Semantics and the Use of Fuzzy Cognitive Maps in Social Sciences. Soft Computing in the Humanities and Social Science, vol. 214, pp. 6-19.

[10] Ketipi, M. K., Koulouriotis, D. E., Karakasis, E. G., Papakostas, G. A. and Tourassis, V. D. (2010). A Flexible Nonlinear Approach to Represent Cause-effect Relationships in FCMs. J. of Applied Soft Computing 12(12): 3757-3770.

[11] Stach, W., Kurgan, L., Pedrycz, W. and Reformat, M. (2005). Genetic Learning of Fuzzy Cognitive Maps. J. of Fuzzy Sets and Systems, 153(3): 371-401.

[12] Stylos, C. D., Georgopoulos, V. C. and Groumpos, P. P. (1997). The Use of Fuzzy Cognitive Maps in Modelling Systems. In Proceedings of 5th IEEE Mediterranean Conf. on Control and Systems, Paphos, Cyprus.

[13] A. Demirbas, "Waste Management, Waste Resource Facilities and Waste Conversion Processes", Energy Conservation and Management 52, 1280-1287, (2011).

[14] M. L. M. Graymore, N. G. Sipe, R. E. Rickson, "Regional Sustainability: How Useful are Current Tools of Sustainability
Assessment at the Regional Scale?", Ecological Economics, Volume 67, Issue 3, 362-372, (2008).

[15] A. van de Klundert, J. Anschutz, "Integrated Sustainable Waste Management: the Selection of Appropriate Technologies and the Design of Sustainable Systems is Not (Only) a Technological issue", CEDARE/IETC Inter-regional Workshop on Technologies for Sustainable Waste Management, Alexandria, Egypt, 1-17, (1999).

[16] A. J. Morrissey, J. Browne, "Waste Management Models and Their Application to Sustainable Waste Management", Waste Management 24, 297-308, (2004)

[17] E. J. Wilson, F. R. McDougall, J. Willmore, "Euro-Trash: Searching Europe for a More Sustainable Approach to Waste management", Resources Conservation and Recycling 31, 327-346, (2001).

[18] D. J. Langa, C. R. Binder et al., "Material and Money Flows as a Means for Industry Analysis of Recycling Schemes. A Case Study of Regional Bio-Waste Management", Resources, Conservation and Recycling 49, 159-190, (2006).

[19] J. den Boer, E. den Boer, J. Jager, "LCA-IWM: A Decision Support Tool for Sustainability Assessment of Waste Management Systems", Waste Management 27,_1032-1045, (2007).

[20] S. A. Thorneloe, K. Weitz, M. Barlaz, R. K. Ham, "Tools for Determining Sustainable Waste Management Through Application of Life-Cycle Assessment: Update on U.S. Research", Seventh International Waste Management and Landfill Symposium V, 629-636, (1999).

[21] S. A. Thorneloe, K. Weitz, M. Barlaz, R. K. Ham, "Tools for Determining Sustainable Waste Management Through Application of Life-Cycle Assessment: Update on U.S. Research", Seventh International Waste Management and Landfill Symposium V, 629-636, (1999).

[22] E. Papageorgiou, A. Kontogianni, "Using Fuzzy Cognitive Mapping in Environmental Decision Making and Management: A Methodological Primer and an Application", International Perspectives on Global Environmental Change, S. Young (ed.), ISBN: 978-953-307-815-1, InTech, DOI: 10.5772/29375, (2012)

[23] "A guide to the project management body of knowledge (PMBOK ${ }^{\circledR}$ Guide) - Fifth Edition", Project Management Institute, Pennsylvania USA, 2013, pp. 391-413

[24] F. Perrini, A. Tencati, "Sustainability and Stakeholder Management: the Need for New Corporate Performance Evaluation and Reporting Systems", Bus. Strat. Env. 15, 296 - 308, 2006

[25] M. F. Hatwágner, A. Torma, L. T. Kóczy, Parameter dependence of Fuzzy Cognitive Maps' behaviour, submitted to ASCC 2015, unpublished.

[26] Team FME, "Project Stakeholder Management - project skills", 2014

[27] Torma, A., Susniene, D., Hatwágner, F. M., Kóczy, T. L.,"A comparative analysis of Stakeholder Management by using FCM", submitted to ICCMIT 2015, unpublished. 